\title{
Prevalence, Management, and Outcome of Atrial Fibrillation and Other Supraventricular Arrhythmias in COVID-19 Patients
}

Michele Magnocavallo, MD ${ }^{a, b}$, Giampaolo Vetta, $\mathrm{MD}^{\mathrm{b}}$, Domenico G. Della Rocca, MD, PhD ${ }^{a, *}$, Carola Gianni, MD, PhD ${ }^{a}$, Sanghamitra Mohanty, MDa, Mohamed Bassiouny, MDa, Luca Di Lullo, MD, PhD ${ }^{c}$, Armando Del Prete, MD ${ }^{d}$, Donatello Cirone, $\mathrm{MPH}^{\mathrm{e}}$, Carlo Lavalle, $\mathrm{MD}^{\mathrm{b}}$, Cristina Chimenti, MD, PhD ${ }^{\mathrm{b}}$, Amin Al-Ahmad, $\mathrm{MD}^{\mathrm{a}}$, J. David Burkhardt, $\mathrm{MD}^{\mathrm{a}}, \mathrm{G}$. Joseph Gallinghouse, $\mathrm{MD}^{\mathrm{a}}$, Javier E. Sanchez, MDa, Rodney P. Horton, MD ${ }^{a}$, Luigi Di Biase, MD, PhD; ${ }^{a, f}$, Andrea Natale, $\mathrm{MD}^{\mathrm{a}, \mathrm{g}, \mathrm{h}}$

\section{KEYWORDS}

- COVID-19 • Supraventricular arrhythmias • Atrial fibrillation • Catheter ablation • Atrial flutter

- Rhythm control

\section{KEY POINTS}

- Supraventricular arrhythmias are common in COVID-19 patients, especially in critically ill.

- Arrhythmias occur after direct viral damage, but also due to systemic involvement.

- Atrial fibrillation represents the most common supraventricular arrhythmias and it is independently associated with in-hospital mortality.

- Supraventricular arrhythmias will be safely treated, minimizing exposure and paying attention to general clinical conditions.

\section{INTRODUCTION}

Severe acute respiratory syndrome coronavirus 2 (SARS-COV-2) is the causative agent of coronavirus disease 2019 (COVID-19); it was officially detected for the first time in Wuhan and has spread throughout the world becoming a pandemic. ${ }^{1,2}$
Although COVID-19 causes respiratory symptoms in most patients, several studies showed an extrapulmonary involvement, including the cardiovascular system. ${ }^{3-5}$ COVID-19 patients may be affected by myocarditis, thromboembolic events, heart failure and cardiogenic shock, acute coronary syndromes,

M. Magnocavallo and G. Vetta equally contributed to the article.

a Texas Cardiac Arrhythmia Institute, St. David's Medical Center, 3000 N. IH-35, Suite 720, Austin, TX 78705 USA; ${ }^{b}$ Department of Cardiovascular/Respiratory Diseases, Nephrology, Anesthesiology, and Geriatric Sci-

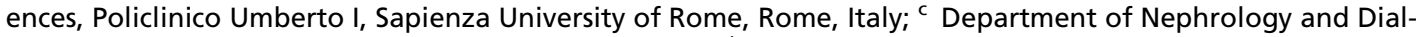
ysis, L. Parodi - Delfino Hospital, Colleferro, Roma, Italy; ${ }^{d}$ Division of Cardiology, Santa Maria Goretti Hospital, Latina, Italy; ${ }^{e}$ Azienda Ospedaliero-Universitaria Careggi, Florence, Italy; ${ }^{f}$ Albert Einstein College of Medicine at Montefiore Hospital, New York, NY, USA; ${ }^{g}$ Interventional Electrophysiology, Scripps Clinic, La Jolla, CA, USA; ${ }^{h}$ Department of Cardiology, MetroHealth Medical Center, Case Western Reserve University School of Medicine, Cleveland, $\mathrm{OH}$, USA

* Corresponding author. Texas Cardiac Arrhythmia Institute at St. David's Medical Center, 3000 North I-35, Suite 720, Austin, TX 78705.

E-mail address: domenicodellarocca@hotmail.it 
and atrial and ventricular arrhythmias. ${ }^{6-8}$ Notably, cardiac arrhythmias occur in $6 \%$ to $17 \%$ of patients, rising to $44 \%$ in patients admitted to the intensive care unit, ${ }^{9}$ resulting the second most frequent complication after acute respiratory distress syndrome. ${ }^{10}$ Several possible mechanisms lead to an increased risk of cardiac arrhythmias during COVID-19 infection, ranging from direct myocardial injury to extracardiac involvement. ${ }^{11}$ Arrhythmias are mainly caused by the hypoxia related to direct viral damage in the lungs, myocarditis, or abnormal inflammatory response and secondarily as a result of myocardial ischemia, myocardial strain, or electrolyte imbalances. ${ }^{11}$ As a matter of fact, arrhythmias are not simply caused by the direct effect of COVID-19 infection, but instead are probably the result of a multifactorial condition. ${ }^{12}$

Supraventricular arrhythmias are the most frequent arrhythmias observed in COVID-19 patients and among them, atrial fibrillation (AF) is the most common occurring in about $15 \%$ to $30 \%$ of them. ${ }^{13}$ The presence of AF is associated with increased clinical manifestations of severe COVID-19 and is independently associated with in-hospital mortality, posing a significant burden to patients, physicians, and health care systems globally. ${ }^{14}$ The complexity of this clinical condition requires a multifaceted and multidisciplinary approach; thus, we provide a comprehensive guidance for monitoring and management of cardiac arrhythmias in COVID-19 patients.

\section{MECHANISMS OF ARRHYTHMOGENESIS IN COVID-19}

COVID-19 infection can lead to an increased risk of cardiac arrhythmias by several pathophysiological mechanisms, which are summarized in Fig. 1. These include different types of myocardial injury and extracardiac processes that may exacerbate arrhythmias in patients with a pre-existing propensity. ${ }^{15}$

\section{Hypoxia}

The most recurrent COVID-19 manifestation is respiratory involvement, which may progress to acute respiratory distress syndrome. Hypoxia results in anaerobic glycolysis causing a decrease of intracellular $\mathrm{pH}$ and electrolyte imbalance, mainly an increase of calcium levels. ${ }^{16} \mathrm{~A}$ higher cytosolic $\mathrm{Ca}^{2+}$ concentration alters cellular action potentials and contribute to the development of early and late afterdepolarizations, which are a known trigger for atrial and ventricular arrhythmias. ${ }^{16}$ Anaerobiosis also results in an increased potassium concentration and, as a consequence, increased cellular excitability and electrical conduction velocity. ${ }^{16}$ In addition, hypoxia reduces electrical coupling and tissue anisotropy via inactivation of connexin-43 in the gap junctions. ${ }^{17}$

Moreover, respiratory failure causes a hyperadrenergic tone, which contributes to the risk of cardiac arrhythmias. ${ }^{18}$ Indeed, hypersympathetic activity leads to an amplified calcium influx into cardiomyocytes, resulting in a calcium overload and frequently delayed afterdepolarizations. ${ }^{19}$

\section{Myocarditis}

Several findings suggest that SARS-CoV-2 is the causative agent of myocarditis and that myocardium involvement may occur by direct virus infection or through infected alveolar macrophages. ${ }^{20,21}$ The virus penetrates the myocardial cell, binding the receptors of the angiotensin-converting enzyme-2 (ACE-2) that will be internalized, leading to a consequent inhibition of angiotensin II degradation. ${ }^{22,23}$ The downregulation of myocardial ACE-2 expression is associated with excessive accumulation of angiotensin II, which causes myocardial injury, remodeling, and even adverse cardiac outcomes. ${ }^{24}$ Thus, downregulation of ACE-2 in COVID-19 might increase AF vulnerability and its perpetuation.

Another potential mechanism is that virusactivated CD8 + T lymphocytes reach the myocardium and can cause myocardial inflammation, as a result of the release of proinflammatory cytokines and activation of T lymphocytes. ${ }^{21}$

In the acute phase of myocarditis, arrhythmogenesis is caused by cellular damage, ionic imbalance, and gap junction dysfunction due to impaired cardiac connexin expression. ${ }^{25}$ In myocarditis, inflammation leads to impaired cellular calcium and potassium homeostasis, producing early and delayed afterdepolarizations and increasing cellular repolarization and conduction time. ${ }^{25}$ Prolonged repolarization time leads to triggered activity, whereas coupling with increased conduction time leads to re-entry circuits. ${ }^{25}$

\section{Myocardial Ischemia}

Myocardial ischemia in COVID-19 patients could be caused by coronary dysfunction and hyperinflammatory response. ${ }^{26}$ The release of cytokines promotes the activation of $\mathrm{T}$ lymphocytes and monocytes within a pre-existing atherosclerotic plaque; the resulting histotoxic effect may cause plaque rupture, thereby leading to an acute coronary syndrome. Moreover, the release of the aforementioned cytokines, specifically interleukin (IL)-6, may exert proatherogenic effects, characterized by vascular smooth muscle proliferation, endothelial cell, and platelet activation. ${ }^{27,28}$ 


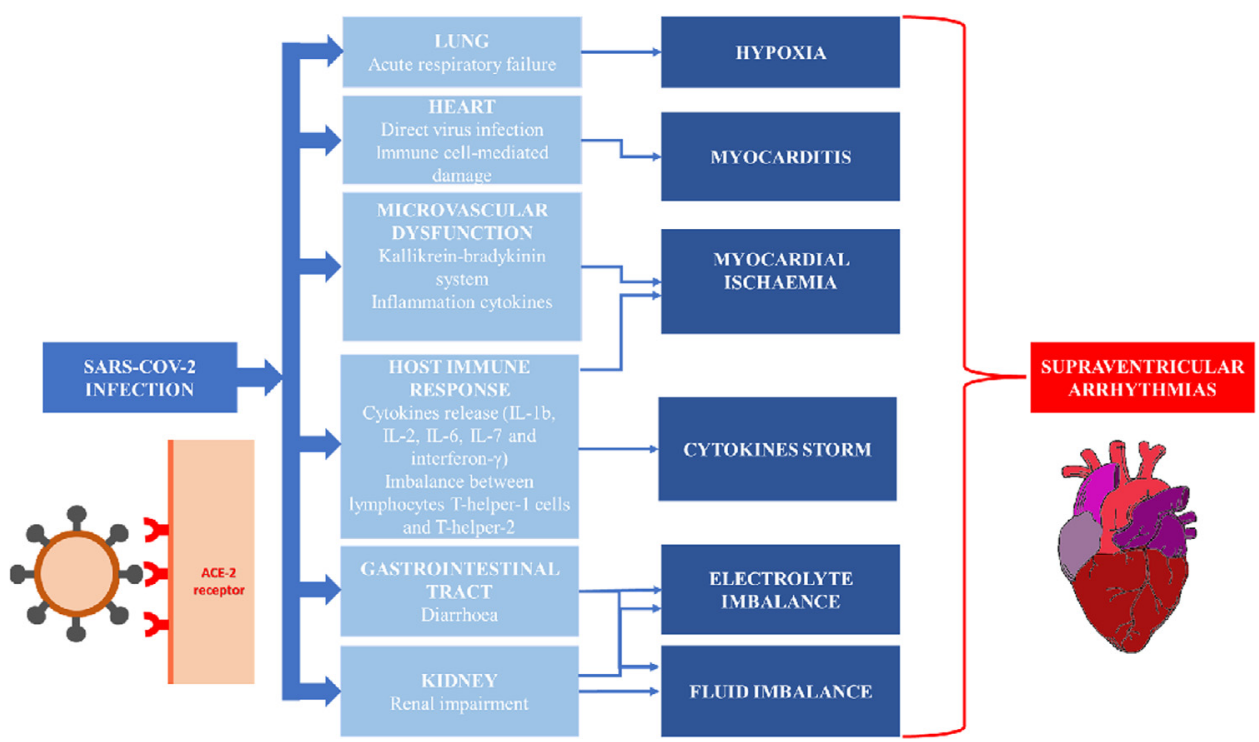

Fig. 1. Potential mechanisms of arrhythmia and COVID-19. IL, interleukin; SARS-CoV-2, severe acute respiratory syndrome coronavirus 2.

Myocardial ischemia can also be caused by microvascular dysfunction due to endothelial impairment. ${ }^{29,30}$ Endothelial dysfunction in COVID-19 patients is caused primarily by the downregulation of ACE-2 receptors, triggering the kallikrein-bradykinin system and resulting in increased vascular permeability. ${ }^{31}$ Neutrophils and T lymphocytes release inflammatory cytokines and vasoactive molecules increasing endothelial cell contractility and vascular permeability.

Virus-mediated vasculitis is another possible mechanism of microvascular dysfunction because the virus penetrates vascular endothelial cells via ACE-2 receptors, leading to inflammation and apoptosis. ${ }^{32}$

\section{Cytokine Storm}

COVID-19 infection causes systemic inflammation and hyperactivation of lymphocytes and monocytes cells, resulting in a cytokine storm (IL-1b, IL-2, IL-6, IL-7, and interferon- $\gamma$ ) and an imbalance between lymphocytes T-helper-1 and T-helper-2 cells. ${ }^{33}$ Distinctive cytokines have been shown to induce AF: tumor necrosis factor- $\alpha$ (TNF- $\alpha$ ) increases AF vulnerability and exerts direct effects on atrial structural and electrical remodeling ${ }^{34-36}$; TNF- $\alpha$ and IL- $1 \beta$ may impair cardiac contractility, which is a known risk factor for arrhythmogenesis; IL-6 reduces cardiac connexins and promotes electrical remodeling during acute inflammation. ${ }^{37,38}$ Furthermore, IL-6, TNF- $\alpha$, and IL-1 can lead to prolongation of the cardiac action potential due to impairment of $\mathrm{K}^{+}$and $\mathrm{Ca}^{2+}$ channels. ${ }^{39}$

\section{Electrolyte Imbalance and Fluid Overload}

Electrolyte abnormalities are a well-known trigger of arrhythmogenesis. ${ }^{40}$ In a study of 416 hospitalized patients with COVID-19 infection, $7.2 \%$ of patients had electrolyte disturbances, such as hypokalemia, hypomagnesemia, and hypophosphatemia. ${ }^{4}$ In particular, hypokalemia is very frequent in patients with COVID-19, affecting up to $61 \%$ of hospitalized patients. ${ }^{41}$ These electrolyte imbalances were primarily caused by COVID-19-associated diarrhea and renal impairment. ${ }^{4}$ Indeed, in a retrospective study of hospitalized patients with COVID-19 infection, $27 \%$ of patients had acute renal failure. ${ }^{42}$ In addition, SARS-CoV-2 causes downregulation of ACE-2 receptors and thereby reduces the feedback effects of ACE-2 on the renin-angiotensin-aldosterone system. ${ }^{41}$ This leads to increased reabsorption of sodium and water, resulting in increased blood pressure and excretion of potassium. The resulting hypokalemia causes hyperpolarization of the myocardiocytes, predisposing to atrial arrhythmias. ${ }^{43}$

\section{SUPRAVENTRICULAR TACHYCARDIA PREVALENCE AND OUTCOME}

Supraventricular arrhythmias are the most frequent arrhythmias among COVID-19 patients. ${ }^{44,45}$ In a recent worldwide survey, about $18 \%$ of enrolled patients developed any arrhythmias $^{46}$ : most of them were supraventricular arrhythmias (81.3\%), AF representing the most common (61.5\%). In another retrospective study, 
166 patients experienced atrial arrhythmias (15.8\%) and newly diagnosed atrial arrhythmias occurred in 101 patients $(9.6 \%)$, corroborating the central role of virus infection in the pathogenesis of cardiac arrhythmias. ${ }^{14}$

Overall, a recent meta-analysis demonstrated that the occurrence of supraventricular arrhythmias was more frequent in critically ill patients (relative risk: 12.1; 95\% confidence interval, 8.517.3), in particular those treated with invasive mechanical ventilation. ${ }^{47,48}$

The occurrence of supraventricular arrhythmias is associated with worse outcomes. Indeed, hospital admission in the intensive care unit and thromboembolic risk (pulmonary embolism, stroke, or deep vein thrombosis) was higher in COVID-19 patients with atrial arrhythmia than the general population. ${ }^{49}$ Therefore, giving the critical conditions of these patients, it is not unexpected that AF should be considered as an independent predictor of 30-day mortality (adjusted odds ratio: 1.93; $P=.007){ }^{14}$

\section{MANAGEMENT OF SUPRAVENTRICULAR ARRHYTHMIAS}

The correct management of supraventricular arrhythmias has a central role in COVID-19 patients, especially those hospitalized with more severe forms of the disease and whose outcomes strictly depend on hemodynamic stability. Although there are few studies about the treatment of arrhythmia in COVID-19 patients, it is necessary to take particular attention to the paroxysmal features of arrhythmias, drug-drug interactions, and limitation of exposure. ${ }^{50,51}$ Given the overwhelming prevalence of $A F$ and atrial flutter (AFL) in patients with COVID-19, we will focus on the treatment of these arrhythmias.

\section{Rhythm Control}

Patients with hemodynamic instability due to newonset AF and AFL should undergo electrical cardioversion (Fig. 2). The choice for electrical cardioversion inevitably involves the need for personnel at bedside, and the possibility of invasive mechanical ventilation, that would increase the development of viral aerosols. ${ }^{50}$ Intravenous infusion of amiodarone is recommended for rhythm control in critically ill patients. ${ }^{51,52}$ Moreover, we should be aware of the combination of amiodarone with hydroxychloroquine and/or azithromycin, as the benefit of the eventual combination has to be weighed against the arrhythmic risk caused by QT prolongation. ${ }^{50,53}$ All interactions between medications for AF and COVID-19 are summarized in Table 1.
Class IC antiarrhythmic agents should be administered with great caution because of their arrhythmogenic and negative inotropic effect, especially in critically ill COVID-19 patients, who are prone to or have already developed myocarditis and heart failure. ${ }^{54}$ Because of possible increases in plasma concentration of flecainide when co-administered with hydroxychloroquine and lopinavir/ritonavir and/or the potential QT-prolonging effects of these drugs, serial ECG monitoring is recommended before and after initiating drug therapy.

However, the only rhythm control strategy is not sufficient to achieve a long-term benefit in patients with acute respiratory failure, if the other existing comorbidities (eg, hypoxemia, inflammation, electrolyte imbalances, metabolic acidosis, volume overload, increased sympathetic tone, bacterial superinfection) are not properly treated. ${ }^{50,55}$ In stable hospitalized patients with AF, antiarrhythmic drugs (such as sotalol, flecainide, amiodarone, and propafenone) should be discontinued and rate control therapy initiated with beta-blockers (or nondihydropyridine calcium channel blockers, unless contraindicated, with or without digoxin) because these drugs represent a safer option when administered in combination with an antiviral therapy. ${ }^{50}$ The combination of verapamil with hydroxychloroquine should be avoided because both drugs exert a negative effect on sinoatrial and atrioventricular nodes causing bradycardia and conduction disturbances. ${ }^{56}$ Therefore, ECG monitoring for bradycardia and conduction disturbance should be considered. All interactions between medications for AF and COVID-19 are summarized in Table 1.

\section{Anticoagulation Therapy}

In COVID-19 patients, anticoagulation is prescribed according to the $\mathrm{CHA}_{2} \mathrm{DS}_{2}$-VASc score. ${ }^{57}$ It is important to highlight that some drugs for the treatment of COVID-19 infection have significant interactions with direct oral anticoagulants (DOACs; Table 1). ${ }^{58}$ In particular, lopinavir and ritonavir may have interactions with cytochrome P450 CYP3A4 and antimalarial drugs through P-glycoprotein inhibition. ${ }^{59}$ In these cases, DOACs should be avoided to reduce the risk of bleeding. ${ }^{60}$

In general, DOACs should be favored over vitamin K antagonists (VKAs), given their better safety profile and the standard, international normalized ratioindependent dosing modalities. ${ }^{61}$ Indeed, VKAs treatment requires regular monitoring of the international normalized ratio, ${ }^{62}$ increasing contact with medical staff, so VKAs should be used preferably only in patients with mechanical prosthetic valves or antiphospholipid syndrome. ${ }^{62}$ Heparins have no 


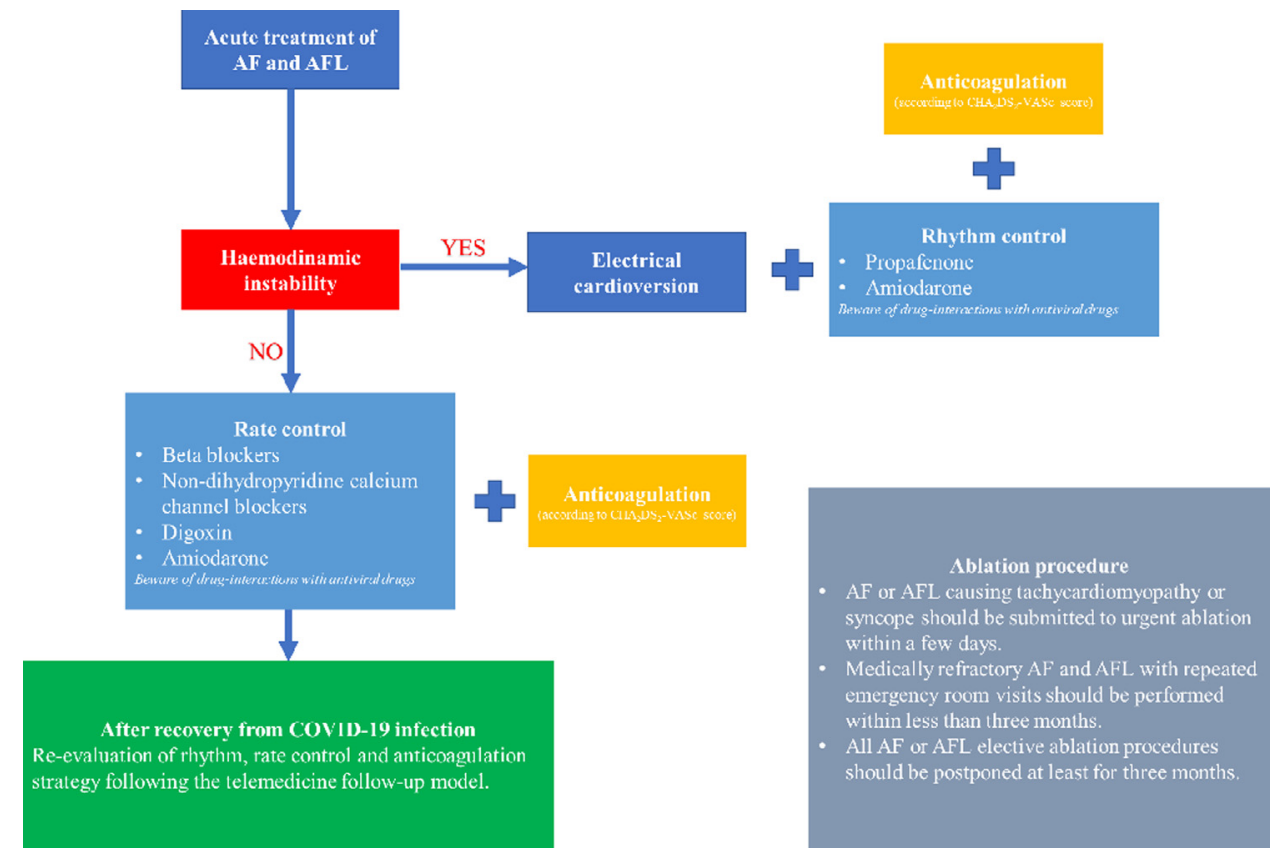

Fig. 2. Acute treatment of $A F$ and $A F L$ in COVID-19 patients. $A F$, atrial fibrillation; $A F L$, atrial flutter.

pharmacologic interactions with drugs for the treatment of COVID-19, making them a safe alternative to oral anticoagulants. Moreover, heparin could provide an anti-inflammatory role in addition to its anticoagulant effect. In fact, heparan sulfate proteoglycans, binding to SARS-CoV-2 spike proteins, decrease host protein binding capability and reduce cytokine cascade. ${ }^{63}$

\section{Echocardiography}

Regarding instrumental examinations in COVID-19 patients, the use of echocardiogram should be limited in order to limit unnecessary contacts among health care providers and patients. ${ }^{50}$ Echocardiography should be performed only if crucial for immediate therapeutic management of critically ill patients. ${ }^{50}$ In this case, transthoracic echocardiography must be chosen over transesophageal echocardiography, thereby avoiding the creation of aerosols. ${ }^{50,64}$ Transesophageal echocardiography should be avoided for early initiation of anticoagulation in new-onset AF, or maintenance of anticoagulant therapy in patients with known AF. ${ }^{65}$ Cardiac computed tomography may be an alternative to transesophageal echocardiography, as it allows ruling out the presence of a left atrial appendage thrombus before cardioversion is performed. ${ }^{66}$

\section{Ablation Procedure}

Catheter ablation for AF patients with an ongoing infection is contraindicated; this same criterion applies to COVID-19 patients. Therefore, both AF or AFL ablation procedures should be postponed to optimize antiarrhythmic therapy and control/ correct all COVID-19 and non-COVID-19-related modifiable risk factors. ${ }^{50,67}$ A different scenario is represented by AF/AFL patients showing evidence of tachycardiomyopathy or syncope; in these patients, an early ablation is pivotal to prevent cardiac remodeling and dysfunction and improve outcomes. ${ }^{68}$ Also, ablation of drug-refractory AF and AFL with repeated emergency room visits should be performed within less than 3 months. ${ }^{50,69-71}$ Of note, universal testing is of utmost importance to create a safe workplace for patients and health care workers, as asymptomatic carriers can be highly contagious and could be unexpectedly admitted. ${ }^{72}$ In case of intubation procedure, this needs to be done out of the electrophysiology laboratory to prevent contamination.

\section{Follow-up in COVID-19 Patients Suffering from Arrhythmias}

The follow-up of patients suffering from arrhythmias must be safe in order to prevent patients from being reinfected by COVID-19. Indeed, as the pandemic progressed, telemedicine has been extensively adopted, ${ }^{73,74}$ allowing face-to-face outpatient appointments to be replaced by teleconsultations. $^{75,76}$ In the TeleCheck-AF project, telemedicine was implemented with remote monitoring of rhythm and frequency of AF, allowing a 


\begin{tabular}{|c|c|c|c|}
\hline Rate Control Drugs & Remdesivir & Hydroxychloroquine & Azithromycin \\
\hline \multicolumn{4}{|l|}{$\underline{\beta \text {-Blockers }}$} \\
\hline Atenolol & - & - & - \\
\hline Bisoprolol & - & - & - \\
\hline Metoprolol & - & - & - \\
\hline Propranolol & - & - & - \\
\hline \multicolumn{4}{|c|}{ Nondihydropyridine calcium channel blockers } \\
\hline Diltiazem & - & - & - \\
\hline Verapamil & - & $\uparrow$ & - \\
\hline \multicolumn{4}{|l|}{ Others } \\
\hline Digoxin & - & $\uparrow \uparrow$ & - \\
\hline \multicolumn{4}{|l|}{ Rhythm control drugs } \\
\hline Amiodarone & - & $\uparrow \uparrow \uparrow$ & $\uparrow \uparrow \uparrow$ \\
\hline Dronedarone & No data available & $\uparrow \uparrow \uparrow$ & $\uparrow \uparrow \uparrow$ \\
\hline Flecainide & - & $\uparrow \uparrow \uparrow$ & $\uparrow \uparrow$ \\
\hline Propafenone & - & - & $\uparrow \uparrow \uparrow$ \\
\hline \multicolumn{4}{|l|}{ Oral anticoagulants } \\
\hline Apixaban & - & $\uparrow$ & $\uparrow \uparrow \uparrow$ \\
\hline Edoxaban & - & $\uparrow \uparrow$ & $\uparrow \uparrow \uparrow$ \\
\hline Rivaroxaban & - & $\uparrow$ & $\uparrow \uparrow$ \\
\hline Dabigatran & - & $\uparrow \uparrow$ & $\uparrow \uparrow$ \\
\hline Warfarin & - & - & - \\
\hline
\end{tabular}

$\uparrow \uparrow \uparrow:$ Potential substantially increased exposure of the medications; these drugs should not be prescribed together.

$\uparrow \uparrow:$ Potential moderately increased exposure of the medications; dosage adjustment or close monitoring may be required.

$\uparrow:$ Potential mildly increased exposure of the medications; the interactions are weak.

-: No significant effects.

complete management of patients, thanks to a mobile phone app using photoplethysmography technology through the built-in camera. ${ }^{77}$ Probably, this model of outpatient management of arrhythmias, thanks to the new wearable technologies, leading to the reduction of the number of hospital visits and health care costs, will remain even after the pandemic and represent an additional weapon in the diagnosis and management of arrhythmias in the near future. ${ }^{78}$

\section{SUMMARY}

Cardiac arrhythmias occur in $6 \%$ to $17 \%$ of COVID19 patients. Their prevalence is significantly higher (up to $44 \%$ ) in patients admitted to intensive care unit, becoming the second most frequent complication after acute respiratory distress syndrome. Supraventricular arrhythmias, mainly AF, are more frequent than ventricular ones. Several mechanisms can contribute to an increased risk of cardiac arrhythmias during COVID-19 infection, ranging from direct myocardial damage to electrolyte imbalance. The main aim of COVID-19-related supraventricular arrhythmia management is to establish a safe treatment plan according to each patient's overall clinical conditions, keep in mind any possible drug-to-drug interactions, and minimize the risk of exposure for the staff and other non-COVID-19 patients.

\section{CLINICS CARE POINTS}

- Arrhythmogenesis is correlated to several pathophysiological mechanisms: hypoxia, myocardial ischemia, inflammation, electrolyte and fluid imbalance.

- Rate Control should be preferred in critically ill COVID-19 patients.- Atrial fibrillation is an indipendent predictor of mortality.

- COVID-19 patients who experienced atrial fibrillation should be monitored to evaluate the burden of arrhythmia. 


\section{DISCLOSURE}

Dr J.D. Burkhardt is a consultant for Biosense Webster and Stereotaxis. Dr L. Di Biase is a consultant for Biosense Webster, Boston Scientific, Stereotaxis, and St. Jude Medical; and has received speaker honoraria from Medtronic, Atricure, EPiEP, and Biotronik. $\operatorname{Dr}$ A. Natale has received speaker honoraria from Boston Scientific, Biosense Webster, St. Jude Medical, Biotronik, and Medtronic; and is a consultant for Biosense Webster, St. Jude Medical, and Janssen. All other authors have reported that they have no relationships relevant to the contents of this article to disclose.

\section{REFERENCES}

1. Lipsitch M, Swerdlow DL, Finelli L. Defining the epidemiology of covid-19 — studies needed. N Engl J Med 2020;382:1194-6.

2. Jee Y. WHO international health regulations emergency committee for the COVID-19 outbreak. Epidemiol Health 2020;42:e2020013.

3. Vetta F. Coronavirus disease 2019 (COVID-19) and cardiovascular disease: a Vicious circle. J Cardiol Cardiovasc Res 2020. https://doi.org/10.37191/ Mapsci-JCCR-1(1)-010.

4. Shi S, Qin M, Shen B, et al. Association of cardiac injury with mortality in hospitalized patients with COVID-19 in Wuhan, China. JAMA Cardiol 2020;5:802.

5. Della Rocca DG, Magnocavallo M, Lavalle C, et al. Evidence of systemic endothelial injury and Microthrombosis in hospitalized COVID-19 patients at different Stages of the disease. J Thromb Thrombolysis 2020. https://doi.org/10.1007/s11239-020-02330-1.

6. Lala A, Johnson KW, Januzzi JL, et al. Prevalence and Impact of myocardial injury in patients hospitalized with COVID-19 infection. J Am Coll Cardiol 2020;76:533-46.

7. Cui S, Chen S, Li X, et al. Prevalence of venous thromboembolism in patients with severe novel coronavirus pneumonia. J Thromb Haemost 2020;18:1421-4.

8. Driggin E, Madhavan MV, Bikdeli B, et al. Cardiovascular considerations for patients, health care workers, and health systems during the COVID-19 pandemic. J Am Coll Cardiol 2020;75:2352-71.

9. Wang D, Hu B, Hu C, et al. Clinical characteristics of 138 hospitalized patients with 2019 novel coronavirus-infected pneumonia in Wuhan, China. JAMA 2020;323:1061.

10. Cheng $P$, Zhu H, Witteles RM, et al. Cardiovascular risks in patients with COVID-19: potential mechanisms and areas of uncertainty. Curr Cardiol Rep 2020;22:34.

11. Dherange $P$, Lang J, Qian $P$, et al. Arrhythmias and COVID-19. JACC: Clin Electrophysiol 2020;6:1193-204.
12. Bhatla A, Mayer MM, Adusumalli S, et al. COVID-19 and cardiac arrhythmias. Heart Rhythm 2020;17: 1439-44.

13. Berman JP, Abrams MP, Kushnir A, et al. Cardiac electrophysiology consultative experience at the epicenter of the COVID-19 pandemic in the United States. Indian Pacing Electrophysiol J 2020;20: 250-6.

14. Peltzer B, Manocha KK, Ying X, et al. Outcomes and mortality associated with atrial arrhythmias among patients hospitalized with COVID-19. J Cardiovasc Electrophysiol 2020;31:3077-85.

15. Xiong T-Y, Redwood S, Prendergast B, et al. Coronaviruses and the cardiovascular system: acute and long-term Implications. Eur Heart J 2020;41: 1798-800.

16. Lazzerini PE, Boutjdir M, Capecchi PL. COVID-19, arrhythmic risk, and inflammation: mind the gap. Circulation 2020;142:7-9.

17. Kolettis TM. Coronary artery disease and ventricular Tachyarrhythmia: pathophysiology and treatment. Curr Opin Pharmacol 2013;13:210-7.

18. Hu H, Ma F, Wei $X$, et al. Coronavirus Fulminant myocarditis treated with glucocorticoid and human Immunoglobulin. Eur Heart J 2021;42:206.

19. Denham NC, Pearman CM, Caldwell JL, et al. Calcium in the pathophysiology of atrial fibrillation and heart failure. Front Physiol 2018;9:1380.

20. Lindner D, Fitzek A, Bräuninger $\mathrm{H}$, et al. Association of cardiac infection with SARS-CoV-2 in confirmed COVID-19 autopsy cases. JAMA Cardiol 2020;5:1281.

21. Siripanthong B, Nazarian S, Muser D, et al. Recognizing COVID-19-related myocarditis: the possible pathophysiology and proposed guideline for diagnosis and management. Heart Rhythm 2020;17: 1463-71.

22. Wrapp D, Wang N, Corbett KS, et al. Cryo-EM structure of the 2019-NCoV spike in the prefusion conformation. Science 2020;367:1260-3.

23. South AM, Diz DI, Chappell MC. COVID-19, ACE2, and the cardiovascular consequences. Am J Physiol Heart Circ Physiol 2020;318:H1084-90.

24. Oudit GY, Kassiri Z, Jiang C, et al. SARS-coronavirus Modulation of myocardial ACE2 expression and inflammation in patients with SARS. Eur J Clin Invest 2009;39:618-25.

25. Tse G, Yeo JM, Chan YW, et al. What is the arrhythmic Substrate in viral myocarditis? Insights from clinical and animal studies. Front Physiol 2016;7:308.

26. Huertas A, Montani D, Savale L, et al. Endothelial cell dysfunction: a major player in SARS-CoV-2 infection (COVID-19)? Eur Respir J 2020;56: 2001634.

27. Madjid M, Vela D, Khalili-Tabrizi H, et al. Systemic infections cause exaggerated local inflammation in atherosclerotic coronary arteries: clues to the 
triggering effect of acute infections on acute coronary syndromes. Tex Heart Inst J 2007;34:11-8.

28. Della Rocca DG, Pepine CJ. Endothelium as a predictor of adverse outcomes: endothelium as a predictor of adverse outcomes. Clin Cardiol 2010;33: 730-2.

29. Musher DM, Abers MS, Corrales-Medina VF. Acute infection and myocardial Infarction. N Engl J Med 2019;380:171-6.

30. Della Rocca DG, Pepine CJ. Some Thoughts on the continuing dilemma of angina pectoris. Eur Heart J 2014;35:1361-4.

31. Pober JS, Sessa WC. Evolving Functions of endothelial cells in inflammation. Nat Rev Immunol 2007; 7:803-15.

32. Varga Z, Flammer AJ, Steiger P, et al. Endothelial cell infection and endotheliitis in COVID-19. Lancet 2020;395:1417-8.

33. Huang C, Wang Y, Li X, et al. Clinical features of patients infected with 2019 novel coronavirus in Wuhan, China. Lancet 2020;395:497-506.

34. Lee S-H, Chen Y-C, Chen Y-J, et al. Tumor Necrosis factor- $\alpha$ alters calcium handling and increases arrhythmogenesis of pulmonary vein cardiomyocytes. Life Sci 2007;80:1806-15.

35. Aschar-Sobbi R, Izaddoustdar F, Korogyi AS, et al. Increased atrial arrhythmia Susceptibility induced by Intense endurance exercise in Mice requires TNF $\alpha$. Nat Commun 2015;6:6018.

36. Mohanty S, Trivedi C, Della Rocca DG, et al. Thromboembolic risk in atrial fibrillation patients with left atrial scar post-extensive ablation. JACC: Clin Electrophysiol 2021;7:308-18.

37. Lazzerini PE, Laghi-Pasini F, Acampa M, et al. Systemic inflammation Rapidly induces Reversible atrial electrical remodeling: the role of interleukin-6-mediated changes in connexin expression. JAHA 2019;8. https://doi.org/10.1161/JAHA.118.011006.

38. Canpolat U, Mohanty S, Trivedi C, et al. Association of Fragmented QRS with left atrial Scarring in patients with persistent atrial fibrillation undergoing $\mathrm{Ra}$ diofrequency catheter ablation. Heart Rhythm 2020; 17:203-10.

39. Puntmann VO, Taylor PC, Barr A, et al. Towards understanding the phenotypes of myocardial involvement in the presence of Self-limiting and Sustained systemic inflammation: a Magnetic Resonance Imaging study. Rheumatology 2010;49:528-35.

40. El-Sherif N, Turitto G. Electrolyte disorders and arrhythmogenesis. Cardiol J 2011;18:233-45.

41. Chen D, Li X, Song Q, et al. Assessment of hypokalemia and clinical characteristics in patients with coronavirus disease 2019 in Wenzhou, China. JAMA Netw Open 2020;3:e2011122.

42. Diao B, Wang C, Wang R, et al. Human Kidney is a Target for novel severe acute respiratory syndrome coronavirus 2 infection. Nat Commun 2021;12:2506.
43. Krijthe BP, Heeringa J, Kors JA, et al. Serum potassium levels and the risk of atrial fibrillation. Int J Cardiol 2013;168:5411-5.

44. Desai AD, Boursiquot BC, Melki L, et al. Management of arrhythmias associated with COVID-19. Curr Cardiol Rep 2021;23:2.

45. Chen Q, Xu J, Gianni C, et al. Simple electrocardiographic criteria for Rapid Identification of Wide QRS complex Tachycardia: the new limb lead algorithm. Heart Rhythm 2020;17:431-8.

46. Coromilas EJ, Kochav S, Goldenthal I, et al. Worldwide survey of COVID-19-associated arrhythmias. Circ Arrhythmia Electrophysiol 2021;14. https://doi. org/10.1161/CIRCEP.120.009458.

47. Goyal P, Choi JJ, Pinheiro LC, et al. Clinical characteristics of covid-19 in New York city. N Engl J Med 2020;382:2372-4.

48. Garcia-Zamora S, Lee S, Haseeb S, et al. Arrhythmias and electrocardiographic findings in coronavirus disease 2019: a Systematic review and meta-analysis. Pacing Clin Electrophysiol 2021;44:1062-74.

49. Zareini B, Rajan D, El-Sheikh M, et al. Cardiac arrhythmias in patients hospitalized with COVID-19: the ACOVID study. Heart Rhythm O2 2021;2:304-8.

50. The European Society for Cardiology. ESC Guidance for the Diagnosis and Management of CV Disease during the COVID-19 Pandemic. Available at: https://www.escardio.org/Education/COVID-19and-Cardiology.

51. Saenz LC, Miranda A, Speranza R, et al. Recommendations for the organization of electrophysiology and cardiac pacing Services during the COVID-19 pandemic: Latin American heart rhythm Society (LAHRS) in collaboration with: Colombian college of electrophysiology, argentinian Society of cardiac electrophysiology (SADEC), Brazilian Society of cardiac arrhythmias (SOBRAC), Mexican Society of cardiac electrophysiology (SOMEEC). J Interv Card Electrophysiol 2020;59:307-13.

52. Goldschlager N, Epstein AE, Naccarelli GV, et al. A practical guide for clinicians who treat patients with amiodarone: 2007. Heart Rhythm 2007;4: 1250-9.

53. Russo V, Carbone A, Mottola FF, et al. Effect of Triple combination therapy with lopinavir-ritonavir, azithromycin, and hydroxychloroquine on QT Interval and arrhythmic risk in hospitalized COVID-19 patients. Front Pharmacol 2020;11:582348.

54. Lavalle C, Trivigno S, Vetta G, et al. Flecainide in ventricular arrhythmias: from old Myths to new perspectives. JCM 2021;10:3696.

55. Ganatra S, Dani SS, Shah S, et al. Management of cardiovascular disease during coronavirus disease (COVID-19) pandemic. Trends Cardiovasc Med 2020;30:315-25.

56. Capel RA, Herring N, Kalla M, et al. Hydroxychloroquine reduces heart rate by Modulating the 
hyperpolarization-activated current if: novel electrophysiological Insights and therapeutic potential. Heart Rhythm 2015;12:2186-94.

57. Hindricks G, Potpara T, Dagres N, et al. 2020 ESC guidelines for the diagnosis and management of atrial fibrillation developed in collaboration with the European association for cardio-Thoracic Surgery (EACTS). Eur Heart J 2021;42:373-498.

58. Agarwal S, Agarwal SK. Lopinavir-Ritonavir in SARSCoV-2 infection and drug-drug interactions with cardioactive medications. Cardiovasc Drugs Ther 2021;35:427-40.

59. Testa S, Prandoni P, Paoletti O, et al. Direct oral anticoagulant plasma levels' Striking increase in severe COVID-19 respiratory syndrome patients treated with antiviral agents: the cremona experience. J Thromb Haemost 2020;18:1320-3.

60. Gawałko M, Kapłon-Cieślicka A, Hohl M, et al. COVID-19 associated atrial fibrillation: Incidence, putative mechanisms and potential clinical Implications. IJC Heart \& Vasculature 2020;30:100631.

61. Lavalle C, Di Lullo L, Bellasi A, et al. Adverse drug Reactions during Real-life use of direct oral anticoagulants in Italy: an update Based on data from the Italian national pharmacovigilance network. Cardiorenal Med 2020;10:266-76.

62. Holbrook A, Schulman S, Witt DM, et al. Evidencebased management of anticoagulant therapy: antithrombotic therapy and prevention of thrombosis, 9th ed: American college of chest physicians evidence-Based clinical practice guidelines. Chest 2012;141:e152S-84S.

63. Yu M, Zhang T, Zhang $W$, et al. Elucidating the interactions between heparin/heparan Sulfate and SARSCoV-2-related proteins - an important strategy for developing novel therapeutics for the COVID-19 pandemic. Front Mol Biosci 2021;7:628551.

64. Irons JF, Pavey W, Bennetts JS, et al. COVID-19 safety: aerosol-generating procedures and cardiothoracic Surgery and anaesthesia - Australian and New Zealand consensus Statement. Med J Aust 2021:214:40-4.

65. Gedikli Ö, Mohanty S, Trivedi C, et al. Impact of dense "Smoke" detected on Transesophageal echocardiography on stroke risk in patients with atrial fibrillation undergoing catheter ablation. Heart Rhythm 2019;16:351-7.

66. Pathan F, Hecht H, Narula J, et al. Roles of Transesophageal echocardiography and cardiac computed tomography for evaluation of left atrial thrombus and associated pathology: a review and critical analysis. JACC Cardiovasc Imaging 2018;11: 616-27.

67. Calkins H, Hindricks G, Cappato R, et al. 2017 HRS/ EHRA/ECAS/APHRS/SOLAECE expert consensus Statement on catheter and Surgical ablation of atrial fibrillation. Europace 2018;20:e1-160.
68. Della Rocca DG, Santini L, Forleo GB, et al. Novel perspectives on arrhythmia-Induced cardiomyopathy: pathophysiology, clinical manifestations and an update on invasive management Strategies. Cardiol Rev 2015;23:135-41.

69. Della Rocca DG, Mohanty S, Mohanty P, et al. Longterm outcomes of catheter ablation in patients with longstanding persistent atrial fibrillation lasting less than 2 Years. J Cardiovasc Electrophysiol 2018;29: 1607-15.

70. Della Rocca DG, Di Biase L, Mohanty S, et al. Targeting non-pulmonary vein triggers in persistent atrial fibrillation: results from a prospective, Multicentre, observational Registry. EP Europace 2021. https://doi.org/10.1093/europace/euab161. euab161.

71. Della Rocca DG, Tarantino N, Trivedi C, et al. Nonpulmonary vein triggers in nonparoxysmal atrial fibrillation: Implications of pathophysiology for catheter ablation. J Cardiovasc Electrophysiol 2020;31: 2154-67.

72. Mohanty S, Lakkireddy D, Trivedi C, et al. Creating a safe workplace by universal testing of SARS-CoV-2 infection in asymptomatic patients and healthcare workers in the electrophysiology units: a Multicenter experience. J Interv Card Electrophysiol 2020. https://doi.org/10.1007/s10840-020-00886-9.

73. Forleo GB, Tesauro M, Panattoni G, et al. Impact of continuous Intracardiac ST-Segment monitoring on Mid-term outcomes of ICD-Implanted patients with coronary artery disease. Early results of a prospective comparison with conventional ICD outcomes. Heart 2012;98:402-7.

74. Della Rocca DG, Albanese M, Placidi F, et al. Feasibility of automated detection of Sleep apnea using Implantable pacemakers and defibrillators: a comparison with Simultaneous polysomnography Recording. J Interv Card Electrophysiol 2019;56:327-33.

75. Piro A, Magnocavallo M, Della Rocca DG, et al. Management of cardiac Implantable electronic device follow-up in COVID-19 pandemic: lessons learned during Italian lockdown. J Cardiovasc Electrophysiol 2020;31:2814-23.

76. Magnocavallo M, Bernardini A, Mariani MV, et al. Home delivery of the communicator for remote monitoring of cardiac Implantable devices: a Multicenter experience during the covid-19 lockdown. Pacing Clin Electrophysiol 2021;44:995-1003.

77. Gawałko M, Duncker D, Manninger M, et al. The European TeleCheck-AF project on remote app-Based management of atrial fibrillation during the COVID19 pandemic: centre and patient experiences. Europace 2021;23:1003-15.

78. Caillol T, Strik M, Ramirez FD, et al. Accuracy of a Smartwatch-derived ECG for diagnosing Bradyarrhythmias, Tachyarrhythmias, and cardiac ischemia. Circ Arrhythmia Electrophysiol 2021;14. 